This item was submitted to Loughborough's Research Repository by the author.

Items in Figshare are protected by copyright, with all rights reserved, unless otherwise indicated.

\title{
Adaptive resource allocation within three-stage OFDM relay networks
}

PLEASE CITE THE PUBLISHED VERSION

PUBLISHER

(C) IEEE

VERSION

VoR (Version of Record)

LICENCE

CC BY-NC-ND 4.0

\section{REPOSITORY RECORD}

Eltayeb, Nasr E.M., Shakiru K. Kassim, and Jonathon Chambers. 2019. "Adaptive Resource Allocation Within Three-stage OFDM Relay Networks”. figshare. https://hdl.handle.net/2134/5381. 
This item was submitted to Loughborough's Institutional Repository (https://dspace.lboro.ac.uk/) by the author and is made available under the following Creative Commons Licence conditions.

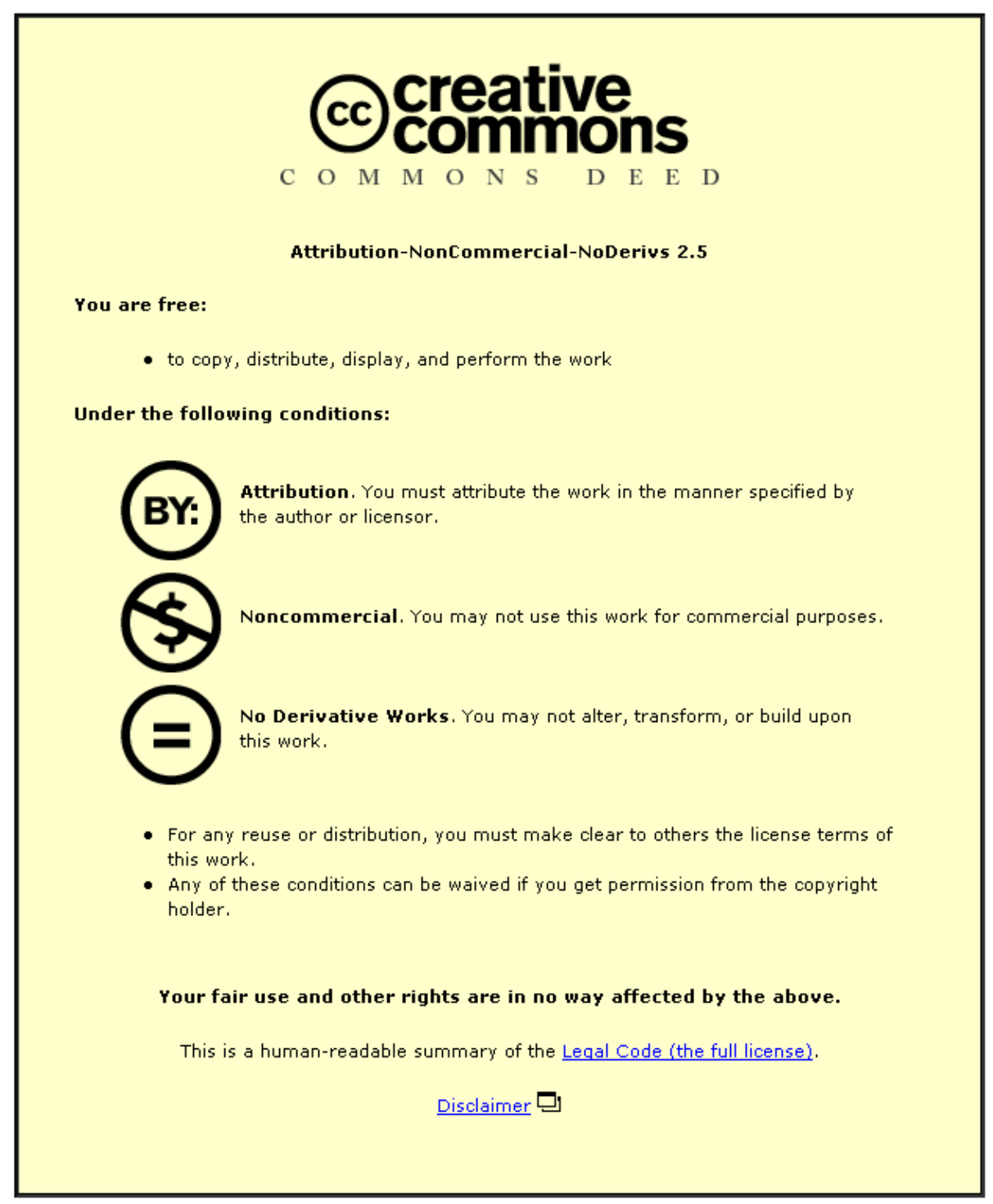

For the full text of this licence, please go to: http://creativecommons.org/licenses/by-nc-nd/2.5/ 


\title{
Adaptive Resource Allocation Within Three-Stage OFDM Relay Networks
}

\author{
N. M. Eltayeb, S. K. Kassim, and J. A. Chambers \\ Advanced Signal Processing Group, Department of Electronic \& Electrical Engineering, \\ Loughborough University, Leicestershire, LE11 3TU, UK. \\ Email: n.e.eltayeb, s.k.kassim, j.a.chambers\}@lboro.ac.uk
}

\begin{abstract}
In this work we consider OFDM transmission, due to its potential for meeting the stringent quality of service $(\mathrm{QoS})$ targets of nextgeneration broadband distributed wireless networks, over three-stage relay networks. In particular, we examine distributed adaptive spacefrequency coding for generally asynchronous links composed of four transmit and/or receive antennas, i.e. exploiting quasi-orthogonal and extended-orthogonal coding schemes. The successful deployment of these closed-loop methods is dependent upon channel state information (CSI) being available for each stage of the network. Taking the maximum endto-end data rate as the optimal criterion, an adaptive resource allocation (RA) scheme suitable for a wide range of signal-to-noise-ratios (SNRs) and a prescribed transmit power budget is proposed to distribute appropriate resources to each stage based on the channel state information (CSI) and knowledge of the network topology.
\end{abstract}

\section{INTRODUCTION}

Orthogonal frequency division multiplexing (OFDM) is a promising technique to achieve high-bit-rate communication systems in frequency selective fading channels, which can eliminate the multi-path effect and inter-symbol-interference (ISI) [1]. But other techniques are required to solve another two outstanding problems: one is power saving for extra-high data rate transmission with quality of service (QoS) assurance; the other is the vulnerable radio propagation since the spectrum for $4 \mathrm{G}$ system will be above $2 \mathrm{GHz}$ [2]. To solve these problems, relaying techniques have been launched, which take advantage of relay stations to obtain additional diversity gain and reduce path loss effects. Their merits have been presented in many materials, and they are considered as an important component for $4 \mathrm{G}$ systems [3].

In relaying techniques there are two principle modes of operation, namely amplify and forward (AF) and decode and forward (DF). Although the DF scheme is more computational complex, it has increased robustness to channel uncertainties and therefore this approach is adopted in this paper.

The OFDM technique is also regarded as a leading candidate for the future generation of cellular network [3][4], which will carry heterogeneous services such as voice (VoIP), video (DVB-H, TV mobile, etc.), gaming (strategy, first person shooter, etc.) and others. It enables very flexible strategies to perform resource allocation, especially for downlink transmissions. Consequently in this paper, we introduce the design of optimum resource allocation algorithms for adaptive relaying transmissions in an OFDM system.

Performance of an OFDM-based relaying system can be enhanced when combined with link adaptation technique, which adjusts link parameters of transmission on all sub-carriers, so as to improve throughput while satisfying bit error rate (BER) requirement. Adaptation in both power and channel statistics (delay-spread) relates to the optimum fractional transmission power allocated to each stage and type of operational modes. In this paper, we propose adaptation to channel statistics by switching to either space frequency code (SFC) or space-time-frequency code (STFC) according to the channel condition as an effective technique of minimizing the receiver error performance degradation due to the highly frequency selective nature of the channel, wherein the channel state information (CSI) is available to the relay nodes. We quantify the error performance degradation of orthogonal block codes (OBC) in fading channels using a fading interference metric, which can be used to select the best space-diversity transmission mode.

This paper is organized as follows: The system model of a cooperative relay network applying STBCs is derived in Section II. The basic principle of Q-OSTBC and E-OSTBC with feedback rotation is described in Section III. Section IV introduces the theoretical performance of optimum resource allocation strategy which is confirmed by simulation results in Section V. Section VI finally concludes this work.

\section{System Model}

In this section, the basic system model for cooperative relaying based OFDM system is introduced. Throughout the paper, it is assumed that a direct communication between the source node $N_{t}$ and the destination node $N_{r}$ is not possible.

Consider a network with one source node, one destination node and two set of R relay nodes. This is depicted in Fig.1, where every node is assumed to have a single antenna and it is half duplex constrained.

The transmission of information from the source node to the destination takes place in three stages. In the first stage, the source broadcasts the information to the first relay group using $N_{c}$-point OFDM modulation after cyclic prefix insertion whose length is greater than the channel order is performed, while OFDM demodulation with $C P$ removal is performed at the destination.

The channel gains are frequency-selective fading with the channel order $L(\mathrm{~L}+1$ taps). All the channels are independent identically distributed (i.i.d). The $l$-th tap channel complex impulse response from the $s$ source to the $i$-th relay is $h_{i}^{s r}(l)$, from the $i$-th relay to the $j$-th relay is $h_{i, j}^{r r}(l)$ and that from the $j$-th relay to the $d$ destination is $h_{j}^{r d}(l)$.

The relay nodes receive the faded and noise corrupted OFDM symbols, processes them and transmits them to the second group relay nodes in the second stage. Likewise, the second group relay nodes, receives the faded and noise corrupted symbols, process them and transmit them to the destination in the third stage. The relay nodes are assumed to have perfect carrier synchronization. We assume that the channel gains $h_{i}^{s r}(l), h_{i, j}^{r r}(l), i, j \in\{1 \cdots, R\}$ are perfectly known by the relay nodes in the first and second stages respectively. The destination node is assumed to have the knowledge of all the channel fading gains $h_{i}^{s r}(l), h_{i, j}^{r r}(l), h_{j}^{r d}(l), i, j, \in\{1, \cdots, R\}$. We assume the channels are quasi-static,i.e., the channels are constant during one OFDM block and change independently from one block to another, and modelled by independent and complex Gaussian distributed random variables with zero mean and unit variance $\sim \mathcal{C}(0,1)$. 


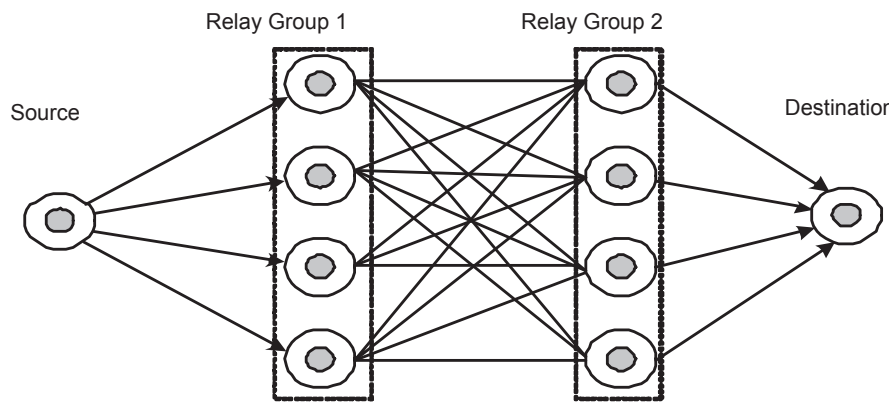

Fig. 1. Three-stage wireless relay network

Let us denote the channel transfer function of the subcarrier $p$ from the $s$ source to the $i$-th relay as $H_{i}^{s r}(p)$, from the $i$-th relay to the $j$-th relay as $H_{i j}^{r r}(p)$ and denote the channel transfer function of the subcarrier $p$ from the $\mathrm{j}$-th relay to the destination as $H_{j}^{r d}(p)$. With these notations, $H_{i}^{s r}(p)=\sum_{l=0}^{L} h_{i}^{s r}(l) e^{-j 2 \pi l p / N_{c}}, H_{i j}^{r r}(p)=$ $\sum_{l=0}^{L} h_{i j}^{r r}(l) e^{-j 2 \pi l p / N_{c}}$ and $H_{j}^{r d}(p)=\sum_{l=0}^{L} h_{j}^{r d}(l) e^{-j 2 \pi l p / N_{c}}$.

At the source, $\mathrm{N}$ symbols $s(p), p=1, \cdots, N$, are transmitted to the first relay group, such that the received signal at the first $i$-th group of relays

$$
y_{i}^{r}=H_{i}^{s r}(p) s(p)+w_{i}(p),
$$

where $w_{i}(p)$ is an independent complex white Gaussian noise with variance $\sigma_{R}^{2}$. Maximum likelihood decoding is then applied across the relays and new vector for transmission by the relays is formed as $y_{i}=$ $\left[y_{1}[1], \cdots, y_{1}[N]\right]$. This block of data is then encoded either over time or frequency to achieve STBC or SFBC transmission between the first and second relay groups. Similar process is performed from the second relay group to the destination.

where $s(p)$ is a unit-energy symbol transmitted from the source and $w_{i}(p)$ is an independent complex white Gaussian noise at the $i$-th relay. The noise variance at all relays is assumed to be $\sigma_{R}^{2}$.

\section{OVERVIEW OF QUASI AND EXTENDED-ORTHOGONAL BLOCK CODES}

\section{A. Quasi-Orthogonal Design}

In this section, the principle of QOBCs with feedback rotation, which are later used as distributed codes in a cooperative relay network, is derived starting [5]. Assuming $T$ orthogonal time intervals and $M$ transmit antennas, an orthogonal design for $N$ complex symbols $x(1), x(2), \cdots, x(N)$ is defined by a code matrix $\mathbf{C}(x(1), x(2), \cdots, x(n))$ of dimension $T \times M$, with $T M \geq N$, such that:

- the entries of $\mathbf{C}$ are complex linear combinations of $x(1), x(2), \cdots, x(N)$ and their complex conjugates $x(1)^{*}, x(2)^{*}, \cdots, x(N)^{*}$.

- $\mathbf{C}^{H} \mathbf{C}=\left(\sum_{n=1}^{N}|x(n)|^{2}\right) \mathbf{I}_{M}$.

where [.] $]^{H}$ designates the conjugate transpose and $\mathbf{I}_{M}$ is an $M \times M$ identity matrix. The symbol transmission rate of these code is defined as $N / T$, i.e., $N$ symbols are transmitted during $T$ time intervals. OSTBCs achieve full diversity order for spatially uncorrelated MIMO channels and can be decoded with a simple maximum likelihood (ML) approach at the receiver [5]. As each symbol $x(n), n=$
$1, \ldots, N$, can be decoded separately, the decoding complexity increases linearly with the code size $N$ and not exponentially as in the case of joint decoding. However, assuming complex transmit symbols there exists no OSTBC for more than two transmit antennas which achieves a symbol transmission rate of one. In general, there exists always a code of rate $1 / 2$, and in particular, there are codes of maximum rate $3 / 4$ for the cases of three and four transmit antennas [5][6].

A symbol transmission rate of one can be achieved by relaxing the orthogonality constraint [5]. Assuming four transmit antennas, the following QOBC with code matrix:

$$
\mathcal{C}=\left[\begin{array}{cccc}
x_{1} & x_{2} & x_{3} & x_{4} \\
-x_{2}^{*} & x_{1}^{*} & -x_{4}^{*} & x_{3}^{*} \\
-x_{3}^{*} & -x_{4}^{*} & x_{1}^{*} & x_{2}^{*} \\
x_{4} & -x_{3} & -x_{2} & x_{1}
\end{array}\right]
$$

is designed [1]. The received signal equation for the QOBC in (2) is given by

$$
\begin{gathered}
{\left[\begin{array}{c}
r_{1} \\
r_{2}^{*} \\
r_{3}^{*} \\
r_{4}^{*}
\end{array}\right]=\left[\begin{array}{cccc}
h_{1} & h_{2} & h_{3} & h_{4} \\
h_{2}^{*} & -h_{1}^{*} & h_{4}^{*} & -h_{3}^{*} \\
h_{3}^{*} & h_{4}^{*} & -h_{1}^{*} & -h_{2}^{*} \\
h_{4} & -h_{3} & -h_{2} & h_{1}
\end{array}\right]\left[\begin{array}{l}
x_{1} \\
x_{2} \\
x_{3} \\
x_{4}
\end{array}\right]+\left[\begin{array}{c}
n_{1} \\
n_{2}^{*} \\
n_{3}^{*} \\
n_{4}
\end{array}\right]} \\
\mathbf{r}=\mathbf{H} \mathbf{x}+\mathbf{n}
\end{gathered}
$$

If $H$ in (4) were orthogonal, i.e., $H^{H} H$ were a diagonal matrix. However, the loss of perfect orthogonality is given by

$$
H^{H} H=\left[\begin{array}{cccc}
\alpha & 0 & 0 & \beta \\
0 & \alpha & -\beta & 0 \\
0 & -\beta & \alpha & 0 \\
\beta & 0 & 0 & \alpha
\end{array}\right]
$$

where

$$
\alpha=\sum_{n=1}^{4}\left|h_{n}\right|^{2}, \quad \text { and } \quad \beta=\operatorname{Re}\left\{h_{1}^{*} h_{4}-h_{2}^{*} h_{3}\right\}
$$

Because of the inter-symbol-interference indicated by variable $\beta$ in (5), the BER performance of this QOBC is degraded. However, from (2) and (5) one notes that the code matrix can be decoupled into two sub-matrices with

$$
\mathbf{C}=\mathbf{C}_{1}(x(1), 0, x(4), 0)+\mathbf{C}_{2}(0, x(2), 0, x(3))
$$

where $\mathbf{C}_{1}^{H} \mathbf{C}_{2}+\mathbf{C}_{2}^{H} \mathbf{C}_{1}=0$ for all $x(n)$. For both symbol pairs $x(1), x(3)$ and $x(2), x(4)$, the ML decoding at the receiver can be processed independently. From the literature, two different approaches for improving the BER performance of QOBCs are proposed. The first approach is proposed to take $x(1)$ and $x(3)$ from different symbol constellation $\mathcal{A}_{1}$ and $\mathcal{A}_{2}=e^{j \phi} \mathcal{A}_{1}$, respectively, by rotating the constellation of $x(3)$ by an angle $\phi$. Similarly, $x(2)$ is taken from $\mathcal{A}_{1}$ and $x(4)$ is taken from $\mathcal{A}_{2}$ for the second symbol pair. By computer search, the optimum rotation angle $\phi_{\text {opt }, 1}$ can be found under the constraint of maximizing the minimum Euclidean distance between all different representations of the symbol pairs $x(1), x(3)$ and $x(2), x(4)$, respectively. For symbols taken from a QPSK constellation, $\phi_{\text {opt }}, 1 \approx 0.525$ maximizes the minimum Euclidean distance. Constellation rotation for QOBCs is also considered in [7]. However, here we consider a feedback method proposed in [1][8] to orthogonalize QOBC by rotating the transmitted signals from certain antennas in a prescribed way based upon the information fed back from the receiver which eliminate the off-diagonal term $\beta$. 
The exploitation of such feedback also permits the use of other better performing block codes.

\section{Extended-Orthogonal Design}

Consider the EOBC for four transmit antennas in [9][10] ( a similar one is presented in [11]), which is given by

$$
\mathbf{C}=\left[\begin{array}{cccc}
x_{1} & x_{1} & x_{2} & x_{2} \\
-x_{2}^{*} & -x_{2}^{*} & x_{1}^{*} & x_{1}^{*}
\end{array}\right]
$$

Using one receive antenna, the received signals for the EOBC in (8) can be expressed by

$$
\begin{gathered}
{\left[\begin{array}{c}
r_{1} \\
r_{2}^{*}
\end{array}\right]=\left[\begin{array}{cc}
h_{1}+h_{2} & h_{3}+h_{4} \\
h_{3}^{*}+h_{4}^{*} & -h_{1}^{*}-h_{2}^{*}
\end{array}\right]\left[\begin{array}{l}
x_{1} \\
x_{2}
\end{array}\right]+\left[\begin{array}{l}
n_{1} \\
n_{2}^{*}
\end{array}\right]} \\
\mathbf{r}=\mathbf{H} \mathbf{x}+\mathbf{n}
\end{gathered}
$$

Applying the matched filtering at the receiver with $H^{H}$ matrix, we can obtain the following matrix

$$
\mathbf{H}^{H} \mathbf{H}=\left[\begin{array}{cc}
\alpha+\beta & 0 \\
0 & \alpha+\beta
\end{array}\right]
$$

where

$$
\alpha=\sum_{n=1}^{4}\left|h_{n}\right|^{2} \quad \text { and } \quad \beta=2 \operatorname{Re}\left\{h_{1} h_{2}^{*}+h_{3} h_{4}^{*}\right\}
$$

provided $\beta$ is positive this will induce improved received signalto-noise ratio over the QOBC scheme in III.

\section{AdAPTIVE SPACE-Frequency CODING}

Wireless channels are generally frequency-selective fading channels. In this context, we propose to spread QOBC and EOBC in time or/and frequency depending on the channel status. The channel status can be categorized into the following scenarios:

- If $B_{c}>\rho \times \Delta f_{\text {sub }}$ and $T_{c}<\rho \times T_{\text {block }}$ then SFBC is used.

- If $T_{c}<\rho \times T_{\text {block }}$ and $B_{c}<\rho \times \Delta f_{\text {sub }}$ then STFBC is used.

where $B_{c}$ and $T_{c}$ are coherence bandwidth and coherence time, $T_{\text {block }}$ is the total OFDM symbol duration including CP and $\rho$ is the number of rows of OBC design. $\Delta f_{s u b}$ is the subcarrier spacing of the OFDM system.

The above means that the $\mathrm{OBC}$ is arranged in two configurations (SFBC and STFBC) depending on the channel condition. In highly frequency and/or time selective channels the block code is spread both in time and/or frequency. In order to realize adaptive time and/or frequency spreading of the $\mathrm{OBC}$, transmitter requires to know the mode switching information [12]. Thus the receiver should be equipped with a channel statistics estimator ( $B_{c}$ and $T_{c}$ estimators) and based on the selection criteria should provide the switching information to the transmitting node through a feedback channel.

\section{Optimum Power allocation Strategy}

In this section, optimum power allocation within the entire network is investigated. Given a fixed power budget, an optimal inter-stage power allocation presented in [13], which minimizes the end-to-end BER with a flat fading inter-stage channels was extended to frequency selective Rayleigh fading channels. Since OFDM is a multi-carrier transmission technique, to further improve the performance of the system, we present an optimal intra-stage power allocation strategy over each sub-carriers in addition to the aforementioned inter-stage.

\section{A. Inter-stage Power allocation Strategy}

If the total power required to deliver information from source to destination is constrained to $P$, this will be distributed among the relaying stages such that

$$
P=\sum_{i=1}^{K} P_{i}
$$

where $K$ is the number of relaying stages and $P_{i}$ is the total power allocated to the $i^{t h}$ stage expressed from [13] as

$$
P_{i}=\left[\alpha_{j}\left(\frac{q_{i}^{-1} A_{i}^{-1} B_{i}^{q_{i}}}{q_{j}^{-1} A_{j}^{-1} B_{j}^{q_{j}}}\right)^{\frac{1}{q_{\max }+1}}\right]^{-1}
$$

where $q_{i}=t_{i} . r_{i}$ which is the product of the number of transmit and received antennas of the $i^{\text {th }}$ stage, $q_{\max }=\arg \max \left(q_{1}, \cdots, q_{k}\right)$ and $j$ is the adjacent relaying stage. The parameter $\alpha_{j}$ is the fractional frame duration which for our TDMA system satisfies $\sum_{i=1}^{K} \alpha_{i}=1$. For the $i^{t h}$ stage fractional frame duration can be expressed as

$$
\alpha_{i}=\frac{\prod_{j=1, j \neq i}^{K} D_{j} \cdot \log _{2}\left(M_{j}\right)}{\sum_{k=1}^{K} \prod_{j=1, j \neq k}^{K} D_{j} \cdot \log _{2}\left(M_{j}\right)}
$$

with $A_{i}$ and $B_{i}$ expressed as

$$
A_{i}=\frac{2 q_{i}}{\log _{2}\left(M_{i}\right)}, \quad \text { and } \quad B_{i}=\frac{G Q A M_{i}}{D_{i}} \frac{\gamma_{i}}{t_{i}} \frac{S}{N}
$$

where $G Q A M=3 / 2 /\left(M_{i}-1\right), \gamma_{i}$ is the total channel gains of the $i^{t h}$ stage, $q_{i}=1-1 / \sqrt{M_{i}}, D_{i}$ is the code rate of the $i^{\text {th }}$ stage while $M_{i}$ is the modulating index and $\frac{S}{N}$ is the $i^{t h}$ stage receiver signal to noise ratio.

\section{B. Intra-stage Power allocation Strategy}

For OFDM transmission the total power $P$ can be distributed as follows:

A. Equal power is allocated to each relaying stage such that each $P_{i}$ is expressed as

$$
P_{i}=\frac{P}{K}
$$

B. As shown in [13], the optimum power allocation strategy that maximizes the capacity and minimizes the end-to-end BER is the one that distributes the power based on the architecture of each stage such that the worst stage (which determines the performance of the entire network) obtains the greater percentage of the total power to improve it performance and hence that of the entire network, hence $P_{i}$ obeys equation (14). However in both A and B equation (13) is satisfied.

Lets $\left\{P_{r}[n]\right\}_{n=1}^{N}$ and $\left\{\lambda_{r}[n]\right\}_{n=1}^{N}$ denote the transmitted power in each sub-carrier and their corresponding channel gain from transmit relay $r$ of the $i^{t h}$ stage respectively. As noted in [3], the well known Shannon's water filling algorithm is the optimal power allocation method for multi-carrier system at low SNR. Here we proposed an explicit approximation of this algorithm which on the average improves the BER performance of the network over a wider range of SNR. The performance of this proposed scheme is compared with equal power allocation to sub carriers.

If $P_{i}[r]$ is the total transmitted power from the $r^{t h}$ relay of the $i^{t h}$ stage, then the power allocated to the $n^{\text {th }}$ sub-carrier is

$$
P_{r}[n]=\left(\frac{\prod_{j=1, j \neq n}^{N} \lambda_{r}[j]^{\frac{-1}{2}}}{\sum_{w=1}^{N} \prod_{j=1, j \neq w}^{N} \lambda_{r}[j]^{\frac{-1}{2}}}\right) P_{i}[r]
$$


If $P_{i}$ is distributed equally among the $R$ transmit relay elements of the $i^{t h}$ stage, we can express the transmitting power on each sub carriers as

$$
P_{r}[n]=\left(\frac{\prod_{j=1, j \neq n}^{N} \lambda_{r}[j]^{\frac{-1}{2}}}{\sum_{w=1}^{N} \prod_{j=1, j \neq w}^{N} \lambda_{r}[j]^{\frac{-1}{2}}}\right) \frac{P_{i}[r]}{R}
$$

therefore

$$
P_{i}=\sum_{r=1}^{R} \sum_{i=1}^{N} P_{r}[n], r \in(1, \ldots, 4)
$$

This power allocation scheme is designed for quasi-static channels.

\section{END-TO-END BIT ERROR RATE}

If $i^{\text {th }}$ relaying stage experience independent probability of error (BER), which is denoted here as $P_{b, i \in(1, K)(e)}$ caused by independent SERs $P_{s, i \in(1, K)(e)}$. The probability of error free transmission at stage $i$ can be expressed as

$$
1-P_{b, i \in(1, K)(e)}
$$

hence the average probability of correct end-to-end transmission $P_{c, e 2 e}(e)$ can be expressed as the joint probability of correct transmission at each stage, i.e.

$$
P_{c, e 2 e}(e)=\prod_{i=1}^{K}\left(1-P_{b, i(e)}\right)
$$

A bit transmitted from a source terminal is received correctly at the destination only when at all the stages the bit has been transmitted correctly.

Thus, the end-to-end BER $P_{b, e 2 e}(e)$ can therefore be expressed as

$$
P_{b, e 2 e}(e)=1-P_{c, e 2 e}
$$

which at low BER at each stage can be approximated as

$$
\begin{aligned}
P_{b, e 2 e}(e) & \approx \sum_{i=1}^{K} P_{b, i}(e) \\
& \approx \sum_{i=1}^{K} \frac{P_{s, i}(e)}{\log _{2}\left(M_{i}\right)}
\end{aligned}
$$

These expression confirms that the end-to-end BER is dominated by the weaker stage, which necessitate the need for power allocation across the network.

\section{Simulation}

In this section, we present the performance of the proposed threestage OFDM relay network for the case $N_{t}=N_{r}=1$ for extendedorthogonal and quasi-orthogonal codes under two and five length CSI assumptions. It is assumed that the channels across the network are spatially uncorrelated Rayleigh fading channels. The modulation scheme of the transmitted signal is quadrature phase shift keying (QPSK). In order to evaluate the diversity performance of the system, we plot the average BER against the average SNR. The total power consumed in the whole network for transmission of the symbols is set to be fixed.

Fig. 2 shows the performance comparison of SFBC and STFBC applied to the last link in the network in order to mitigate the error performance rate. As the last link is performing worse compared to the first and second links in this particular network architecture. From the figure we can see that STFBC for extended-orthogonal coded OFDM systems are susceptible to the channel variation at high SNR. Because in the decoder the STFBC assumes that the channel responses are constant during one time slot and across one frequency bin and SFBC assumes that the complex channel gains between adjacent sub-carriers are approximately constant. We observe that there is about a $2-\mathrm{dB}$ degradation at $B E R=10^{-2}$ in the performance of STFBC decoder compared to the performance of the SFBC scheme. This degradation is increased with high SNR. While in Fig. 3, STFBC for quasi-orthogonal has better performance than the SFBC scheme; i.e., SFBC scheme is susceptible to channel variation. This gain is attributed to the assumption that the channel responses are constant during two time slots and across two frequency bins. For example, at a target $B E R=10^{-2}$ about $1-\mathrm{dB}$ gain can be obtained compared to the SFBC scheme performance.

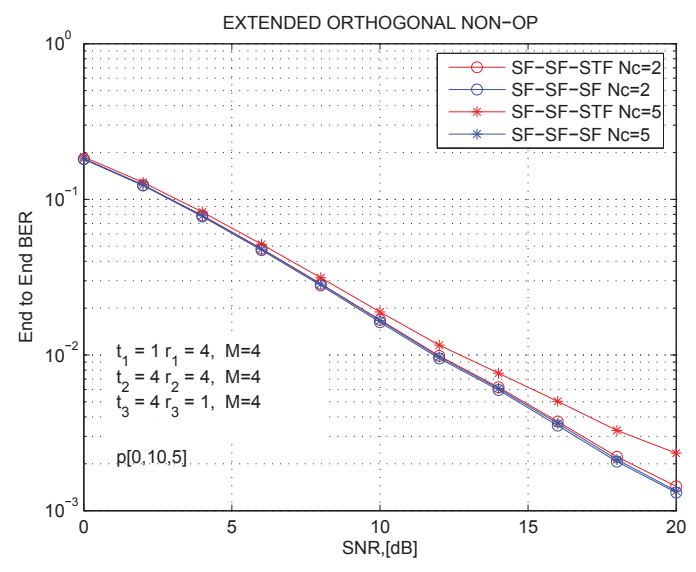

Fig. 2. Performance comparison of BER for Adaptive non-optimum EO-SF and EO-STF coded OFDM system for $L=2$ and $L=5$.

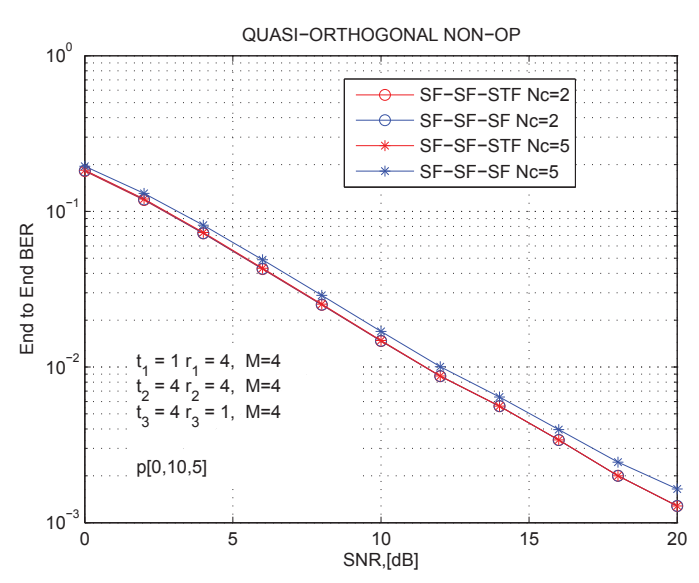

Fig. 3. Performance comparison of BER for Adaptive non-optimum QO-SF and QO-STF coded OFDM system for $L=2$ and $L=5$.

Figs. 4 and 5 compare the optimum power allocation scheme derived in Section VI with the system with equal power allocation among the source and relays for both extended- and quasi-orthogonal respectively. It can be seen that the optimum power allocation scheme considerably preserves the power consumption in the network for achieving the given BER (QoS) at the destination. Those figures illustrate that using the proposed power allocation in this paper a an 


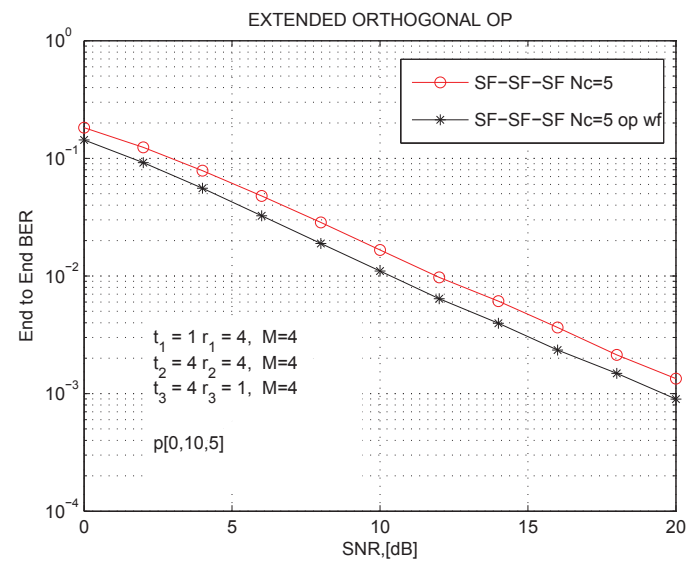

Fig. 4. Performance comparison of BER for Adaptive optimum EO-SFBC for $L=5$.

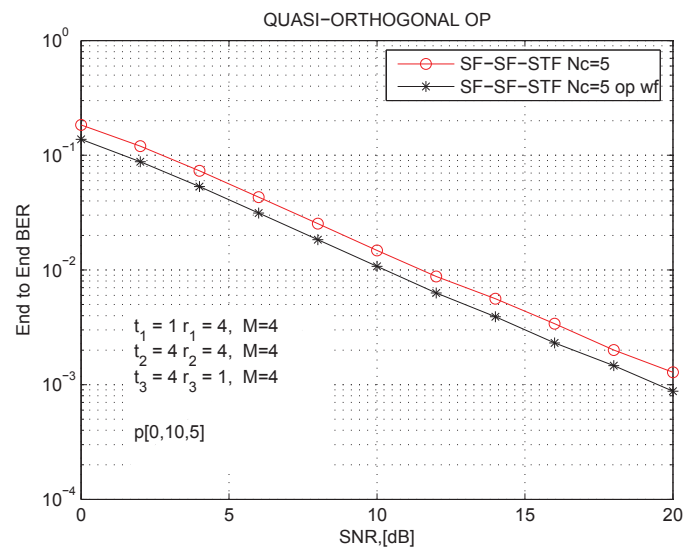

Fig. 5. Performance comparison of BER for Adaptive optimum EO-STFBC for $L=5$.

approximate 2-dB gain in term of network lifetime will be obtained comparing to equal power strategy.

\section{CONCLUSION}

In this paper, we proposed adaptive resource allocation strategies that take both the statistical CSI and the optimum fractional power allocated to each stage into account to improve the performance of the multistage OFDM relay network. Simulations demonstrate that the proposed minimum power allocation strategy could considerably save the total transmitted power compared to equal transmitted power scheme. The proposed scheme can be considered as a promising technique for high data rate wireless transmission over frequency selective multi-path fading channels for broadband wireless communication systems.

\section{REFERENCES}

[1] C. Toker, S. Lambotharan, and J. A. Chambers, "Closed-Loop QuasiOrthogonal STBCs and their Performance in Multipath Fading Environments and When Combined With Turbo Codes," IEEE Trans. Wireless Communications, Vol.3(6), pp.1890-1896, Nov. 2004
[2] L. Hongwu, and L. Ji, "Space-Frequency OFDM System with NullSteering Beamformer," ITS Trans. Information Theory, pp.341-344, June 2006.

[3] J. Shi, G. Yu, Z. Zang and P. Qui, "Resource Allocation in OFDM based Multihop Wireless Networking," in Proc. 63rd IEEE VTC, Vol. 1, pp.319323, May. 2006.

[4] D. Agrawal, V. Tarokh A. Naguib, and N. Seshadri , "Space-Time Coded OFDM for High Data-Rate wireless Commun. Over Wideband Channels,", 48th IEEE VTC Conference, Vol.3, pp.2232-2236, May 1998.

[5] H. Jafarkhani, "A Quasi-Orthogonal Space-Time Block Code," IEEE Trans. Commun., Vol.49, No.1, pp.1-4, Jan. 2001.

[6] V. Tarokh, H. Jafarkhani, and A. R. Calderbank, Space time block codes from orthogonal designs, IEEE Trans. on Information Theory, Vol.45, pp.1456-1467, July 1999.

[7] W. Su and X. G. Xia, "Signal Constellations for Quasi-Orthogonal SpaceTime Block Codes With Full Diversity," IEEE Trans. Information Theory, Vol.50, no.10, pp.2331-2347, Oct. 2004.

[8] S. Lambotharan, and C. Toker, "Closed-loop space time block coding techniques for OFDM based broadband wireless access systems," IEEE Trans. Consumer Electronics, Vol.51, pp.765-769, August 2005.

[9] N. M. Eltayeb, S. Lambotharan, and J. A. Chambers, "A Phase Feedback Based Extended Space-Time Block Code for Enhancement of Diversity," 65th IEEE VTC Conference pp.2296-2299, April 2007.

[10] Y. Yu, S. Keroueden, and J. Yuan, "Closed-Loop Extended Orthogonal Space-Time Block Codes for Three and Four Transmit Antennas," IEEE Signal Processing Letters, Vol.13, No.5, pp. 273-276, May 2006.

[11] J. Akhtar and D. Gesbert, "Extended Orthogonal Block Codes With Partial Feedback," IEEE Trans. Wireless Communications, Vol.3, no.6, pp.1959-1962, Nov. 2004.

[12] [12] J. L. Ong, A. D. S. Jayalath, C. R. N. Athaudage, "Adaptive Time-Frequency Spreading of Quasi-Orthogonal Block Codes for MIMOOFDM Systems," 10th IEEE ICCS Conference, pp.1-7, Oct. 2006.

[13] M. Dohler, "Virtual Antenna Arrays" PhD Thesis, University of London. Nov. 2003. 